\title{
Avenues of discovery in bioprospecting
}

Sir - The experts consulted by Colin Macilwain ${ }^{1}$ provided useful evidence that bioprospecting for natural products is not the most productive way of finding biologically active compounds, but they $\mathrm{did}$ not explain why this should be so.

We would suggest that the high expectations for bioprospecting were partly based on the erroneous belief that a very high proportion of natural products must have some biological activity. However, the Screening Hypothesis ${ }^{2}$, which we proposed to explain natural product chemical diversity, argues that specific, potent biological activity is a rare property for a chemical to possess, irrespective of whether a human, microbe or plant has done the synthesis. This is primarily because potent activity requires a very high degree of 'fit' between the chemical and a target receptor.

The hypothesis proposes that, in organisms that make natural products, there will have been a selection of biochemical traits in natural product biosynthetic pathways which enhance the generation and retention of chemical diversity. Because potent, specific biological activity is an extremely rare property for any molecule to possess, much of this natural product diversity will possess no inherent biological activity. This model proposes that organisms making natural products have been conducting combinatorial biochemistry and have been screening for activity for hundreds of millions of years ${ }^{3}$ before humans adopted a similar strategy. However, most organisms will be screening for biological activity with no particular relevance to human physiology.

So it is predictable that an efficient method of generating chemical diversity (combinatorial chemistry) coupled to highthroughput screening based on a cell process of known importance to human physiology will be more efficient than bioprospecting when searching for drugs.
Many compounds can be screened rapidly even though most will be inactive.

The opportunities that do exist for bioprospecting possibly lie more with the discovery of genes coding for enzymes involved in natural product biosynthesis, many of which might be expected to have a broad substrate tolerance. The addition of such genes to organisms with an existing rich natural product diversity should generate even more chemical diversity, producing chemical structures that currently lie beyond the scope of combinatorial chemistry. This opportunity to build on the existing combinatorial biochemistry of organisms will only be realized if the genetic diversity underlying the synthesis of natural products is explored and understood. So the bioprospecting for chemicals might have been relatively unsuccessful but bioprospecting for genes to use in combinatorial biochemistry might be more successful.

\section{Richard D. Firn}

Department of Biology,

University of York,

York, UK

e-mail:drf1@york.ac.uk

Clive G. Jones

Institute of Ecosystem Studies,

Millbrook, New York 12545, USA

e-mail:clivegjones@ecostudies.org

Sir - An approach to improving the efficiency of identifying pharmaceutical lead compounds from natural products is to analyse and use ethnomedical information to guide the selection of plants. One example is traditional Chinese medicine. The strengths of the approach lie in the fact that Chinese medicine has a long history, was built on a theoretical (rather than purely empirical) basis, and its wealth of clinical experience has been recorded in literature.

In the United States, three new drugs of Chinese-herb origin have recently been developed. They include a derivative of the antimalarial drug qinghaosu, the Alzheimer's drug huperzine $\mathrm{A}^{4}$, and the anti-HIV therapeutic trichosanthin ${ }^{5}$. These drugs may not finally make their way to the US market. But their novel prototype structures and new mechanisms of action have provided scientists with unique inspiration in the search for therapeutic agents.

Despite the vast gap between Chinese and Western medicines, decades of research has revealed that there is a degree of correlation between traditional indications of herbs and their pharmacological effects as defined by Western biomedicine. For instance, many herbs for 'eliminating heat and toxins' in the terms of traditional Chinese medicine possess antibacterial, antiviral, antipyretic and anti-

inflammatory actions. A major mode of pharmacological actions of 'tonics' is to stimulate nonspecific and specific immune functions; and herbs for 'activating blood circulation and removing stasis' can improve blood flow dynamics, exert antithrombosis effect, and improve microcirculation.

The knowledge of such a relationship, which has been documented comprehensively ${ }^{6}$, should prove useful in a natural product drug discovery programme. If a project is set up to identify anti-thrombosis agents, for instance, then herbs used for 'activating blood circulation and removing stasis' ought to be examined. Tianhan Xue \& Li Zhang

154 Kingston Avenue, Suite E,

Santa Barbara,

California 93117, USA

1. Macilwain, C. Nature 392, 535 (1998).

2. Jones, C. G. \& Firn, R. D. Phil. Trans. Roy. Soc. B333, 273 (1991).

3. Firn, R. D. \& Jones, C. G. Recent Advances in Phytochemistry (eds Romeo, J. T. et al.) 295-312 (Plenum, 1996).

4. Borman, S. Chem. \& Eng. News 20 Sept., p. 35 (1993).

5. Byer, V. S. et al. Aids 4, 1189 (1990).

6. Chen, C. (ed.) The methodology for investigation on pharmacology of Chinese herbal medicine (People's Health Publisher, Beijing, 1993).

\section{Smarter than you think}

Sir - Robert W. Old may be overestimating the difficulties students may have with the book Principles of Development by me and others (Nature 392, 325; 1998).

It is to be hoped that they will not read single sentences in isolation from the rest of the text. Just four lines up from the offending sentence referring to Toll receptor activation, we describe Spaetzle binding to Toll. Indeed, on this and on the previous page, and in several figures, it is made clear that Spaetzle is the ligand for the Toll receptor. Students may be more intelligent than Old supposes.

\section{Lewis Wolpert}

Department of Anatomy and Developmental Biology, University College London Medical School, Medawar Building,

Gower Street, London WC1E 6BT, UK

\section{Cancer controversy}

Sir - Your story about Gina Kolata's cancer research article in The New York Times reports a malicious rumour for which there is no evidence (Nature 393, 104; 1998).

For the record, after reading The New York Times article, I called Ms Kolata (which is perfectly proper in my role as her literary agent), she submitted a two-page letterproposal and then she withdrew it. The timing is such that she could not have been colluding with herself to hoodwink The New York Times. Her ethical reputation is among the highest.

John Brockman

Brockman Inc., 5 East 59th Street,

New York, New York 10022, USA

e-mail:john@brockman.com 